\title{
To Combine or Not Combine: Drug Interactions and Tools for Their Analysis. Reflections from the EORTC-PAMM Course on Preclinical and Early-phase Clinical Pharmacology
}

\author{
BTISSAME EL HASSOUNI ${ }^{1}$, GIULIA MANTINI ${ }^{1}$, GIOVANNA LI PETRI ${ }^{1}$, MJRIAM CAPULA ${ }^{2}$, LENKA BOYD $^{1}$, \\ HANNAH N.W. WEINSTEIN ${ }^{1}$, ANDREA VALLÉS-MARTI ${ }^{1}$, MATHILDE C.M. KOUWENHOVEN ${ }^{3}$, ELISA \\ GIOVANNETTI ${ }^{1}$, BART A. WESTERMAN ${ }^{4}$ and GODEFRIDUS J. PETERS ${ }^{1}$, on Behalf of the EORTC PAMM Group \\ ${ }^{1}$ Department of Medical Oncology, Amsterdam UMC, VU University Medical Center, Amsterdam, the Netherlands; \\ ${ }^{2}$ Fondazione Pisana per la Scienza, Pisa, Italy; \\ ${ }^{3}$ Department of Neurology, Brain Tumor Center Amsterdam, \\ Amsterdam University Medical Center, Cancer Center Amsterdam, Amsterdam, the Netherlands; \\ ${ }^{4}$ Department of Neurosurgery, Amsterdam UMC, VU University Medical Center, Amsterdam, the Netherlands
}

\begin{abstract}
Combination therapies are used in the clinic to achieve cure, better efficacy and to circumvent resistant disease in patients. Initial assessment of the effect of such combinations, usually of two agents, is frequently performed using in vitro assays. In this review, we give a short summary of the types of analyses that were presented during the Preclinical and Early-phase Clinical Pharmacology Course of the Pharmacology and Molecular Mechanisms Group, European Organization for Research and Treatment on Cancer, that can be used to determine the efficacy of drug combinations. The effect of a combination treatment can be calculated using mathematical equations based on either the Loewe additivity or Bliss independence model, or a combination of both, such as Chou and Talalay's mediandrug effect model. Interactions can be additive, synergistic (more than additive), or antagonistic (less than additive). Software packages CalcuSyn (also available as CompuSyn) and Combenefit are designed to calculate the extent of the combined effects. Interestingly, the application of machinelearning methods in the prediction of combination treatments, which can include pharmacogenomic, genetic, metabolomic and proteomic profiles, might contribute to
\end{abstract}

Correspondence to: Professor. Dr. G.J. Peters, Department of Medical Oncology, Amsterdam UMC, VU University Medical Center, De Boelelaan 1117, $1081 \mathrm{HV}$ Amsterdam, the Netherlands. Tel: +31 204442633, Fax: +31 204443844, e-mail: gj.peters@vumc.nl

Key Words: Combination treatment, synergy, Calcusyn, Compusyn, review. further refinement of combination regimens. However, more research is needed to apply appropriate rules of machine learning methods to ensure correct predictive models.

Even as early as the 1960s, the majority of clinical treatments consisted of combination regimens. Combinations such as mechlorethamine, vincristine, procarbazine and prednisone (MOPP), and cyclophosphamide, hydroxydaunorubicin and oncovin with prednisone (CHOP) represented a breakthrough in the cure of lymphoma, while other combinations led to a high curation rate in childhood leukaemia (1). Depending on the type of combination used, the treatment rationale is to i) increase the efficacy of each separate drug without increasing toxicity, ii) add a drug which offers protection against toxicity, iii) bypass resistance development, or iv) target different subpopulations in a heterogeneous tumour. The initial clinical rationale was to achieve a better therapeutic effect (e.g. a complete response) than accomplished by each drug separately (e.g. only a partial response) (2). Historically, the selection of drugs to apply in combination therapies was based on the observation that each of the drugs showed antitumor activity against a certain tumour type, preferably with different toxicities of the two drugs. Doses and schedules were determined by trial and error. Soon thereafter, a complementary scientific approach was used to select combinations based on the mechanisms of action of each drug (3). An excellent example is the gemcitabine-cisplatin combination, which was initially developed by our group (4) (with the aim of preventing repair of DNA-platinum adducts) and is now standard therapy for tumours such as non-small cell lung cancer and bladder cancer. Another combination is 
5-fluorouracil (5-FU) and leucovorin (folinic acid) for which we demonstrated in model systems (cell lines, experimental tumours and in tumours of patients) that leucovorin increased and prolonged the inhibition of the 5-FU target, thymidylate synthase (5). 5-FU and leucovorin are part of the standard combination of drugs used in regimens of folinic acid, 5-FU and oxaliplatin (FOLFOX), folinic acid, 5-FU and irinotecan (FOLFIRI) (colon cancer) and FOLFIRI with oxaliplatin (FOLFIRINOX) (pancreatic cancer). The increased toxicity which is often observed with these combinations is usually controlled by combinations with anti-emetics or a corticosteroid such as dexamethasone. The latter may also have (or influence) antitumor effects (6).

Currently most combinations are established using various in vitro assays either focusing on the interaction of drugs on a specific target in a cell-free system, or using a pharmacological assay as summarized previously (7-9). However, an often observed mistake is the lack of proper controls (simply testing the effect of the single agent and combined effect over the whole tested concentration range on cells).

The effect of combination treatment compared to monotherapies can be described as synergistic, additive or antagonistic. The definition is dependent on the mathematical model used, but in general it can be stated that additivity means that the predicted effect of the combination is equivalent to either the sum or the product of each separate effect. Synergism is better than the expected theoretical effect (higher than the sum or lower than the product) and antagonism is worse than the expected theoretical effect (lower than the sum or higher than the product).

In this review, we give a short summary of the types of analyses that can be used to determine the efficacy of drug combinations. Furthermore, we summarize the advantages and disadvantages of these methods and lastly discuss emerging computational approaches.

\section{Methods for Determining the Effect of Drug Combinations}

Several mathematical models were initially used to evaluate drug interactions in cell-free systems, in which the definition of the reference state (meaning no interaction) was the basis. In the Loewe additivity model (10), it was hypothesized that when drug $\mathrm{A}$ is combined with itself, the effect would be the sum of: $A+A=2 A$. When another drug was used, the reference state would be $\mathrm{A}+\mathrm{B}=2 \mathrm{~A}$. In the Bliss independence model (11), which is most commonly used, the reference state (additivity) is a product of the fractional response, in which $0.5 \times 0.5=0.25$. Almost all current models are either a modified use of the Bliss and Loewe models, or are predominantly based on the Bliss model. Application of cellfree models to cellular systems assumes a sigmoidal dose- response curve based on the Hill equation allowing (a) fractional-effect analysis, (b) isobolograms, (c) the response surface area model, based on a mixed Loewe-Bliss; and (d) median effect analysis (Figure 1).

\section{Fractional-effect Analysis}

Fractional-effect analysis determines the theoretical additive effect of a combination by multiplying the effect of each drug alone (12). When drugs A and B are combined at an equitoxic concentration, e.g. achieving 50\% growth inhibition $\left(\mathrm{IC}_{50}\right)$, the theoretical fractional effect is 0.5 for each drug, and additivity is $0.5 \times 0.5=0.25$. When drug $\mathrm{C}$ has a moderate effect, e.g. $25 \%$ growth inhibition at a specific concentration $\left(\mathrm{IC}_{25}\right)$, the fractional effect $(f a)$ is calculated as: $(f a)=(1-\mathrm{g}$ Growth inhibition in \%)/100 resulting in a value of 0.75 . When treatment with drug $\mathrm{D}$ has only a minor effect e.g. $2 \%$ growth inhibition, the fractional effect is 0.98 . The additivity of the combination of drugs $\mathrm{C}(\mathrm{fa}=0.75)$ and $\mathrm{A}(\mathrm{fa}=0.5)$ is equal to their product, i.e. 0.375 , and that for drugs $\mathrm{D}(\mathrm{fa}=0.98)$ and $\mathrm{A}(\mathrm{fa}=0.5)$ is similarly computed to give 0.49. Synergism is achieved for these combinations when the experimentally determined fractional effect is lower than 0.25 (A combined with B), 0.375 (A combined with C) or (A combined with D) 0.49 , respectively. Antagonism is achieved when these values are higher than $0.25,0.375$ or 0.49 , respectively. This method is rather straightforward, but a linear concentration-effect relationship is assumed with sigmoidal dose-response curves. The model does not allow calculation of the variation (confidence interval) within each experiment, only between experiments, and cell kill (a negative fraction) cannot be evaluated.

\section{Isobologram}

The first isobolograms were designed in the 1950s by Nobel laureates Elion and Hitchings (13), in which, for each given level of toxicity, the dose of one drug is plotted on the $\mathrm{x}$ axis and that of the second drug on the y-axis (Figure 1B), e.g. equitoxic doses of the single drugs. When there is additivity, there should be a straight line connecting the plotted $\mathrm{IC}_{50}$ values and when the effect of the combination treatment is synergistic, the plotted line falls to the left of this line and when antagonistic, to the right of this line (14). Subsequently Chou and Talalay (15), computerized the model applying the CalcuSyn program (Biosoft, Cambridge, UK) (see below). Despite the simplicity and accuracy of this method, the extent of synergism or antagonism cannot be quantified, nor is it possible to calculate the variation between experiments, in contrast to fractional-effect analysis. Cell kill cannot be evaluated either. However, this model proved very valuable to move the first combinations into the clinic. 
A

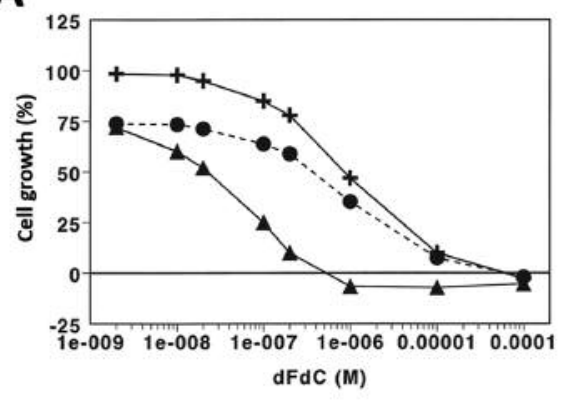

C

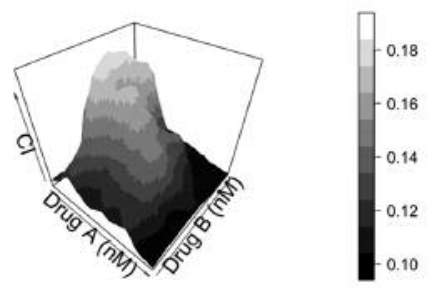

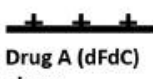

alone

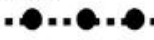

Drug A+B:

predicted when

additive $\left(0.75^{*}+\right)$

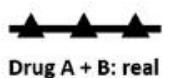

data: synergistic

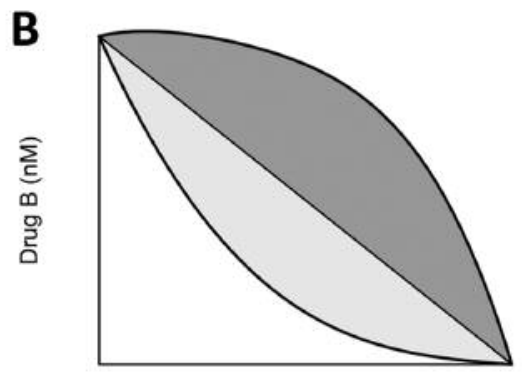

Drug A (nM)

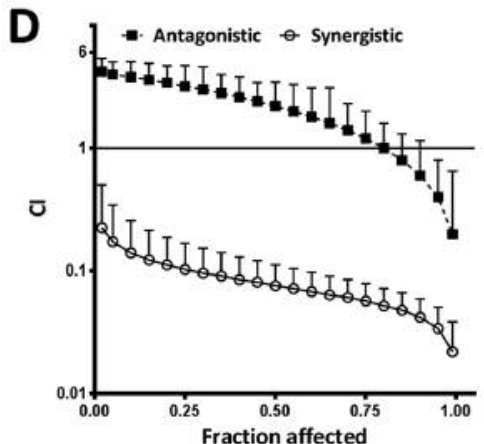

Figure 1. Examples of plots to determine the effect of combination treatments. A: Fractional-effect analysis, reprinted with permission from (3). B: Isobologram. C: The response surface area model (left) and a contour plot (right). D: Median-effect analysis. CI: Combination index.

\section{The Response Surface Area Approach}

According to several mathematicians (14-16), the response surface area model is to be preferred, since this method allows calculation of the extent of synergism/antagonism (called the envelop of synergism), a confidence interval, and the evaluation of more than two drugs. The results of such analysis are presented in various forms, and usually include a 3-D plot or a contour plot. In the 3-D plot, the concentration of the drugs is plotted on two corresponding horizontal axes and the effect of the combination (response) on the vertical axis (Figure $1 \mathrm{C}$, left). In the initial presentations, synergism was considered a combination index (CI) value $>0$ and antagonism as less than 0 . Later presentations of the 3-D plots used different units for the response (i.e. synergy/antagonism), such as a ratio, percentage change or \% relative to the control. Synergism is usually presented as a different colour/peak. An alternative presentation is a contour plot, in which the concentration of a drug is on one of the axes and synergism is shown as a 'contour' of a different colour, either white in black/white presentation, or red or blue according to the preference of the mathematician (Figure 1C, right). Despite ready-to-use mathematical models, evaluation of response surface area plots requires a considerable insight into mathematics. Moreover, the presentation of the plots does not allow for determination of whether synergism is observed under clinically relevant conditions, since the extent of growth inhibition is often not shown and also cell kill is often not considered in the calculation. However, in more recent applications, the so-called Bliss index (a measure for synergy) can be given for each effect of a combination. However, in order to optimally predict the efficacy of the combination, experience with the model and sufficient statistical knowledge is essential.

\section{Median Drug-effect Analysis}

In order to provide a pragmatic and easy-to-use method to evaluate combinations, Chou and Talalay (15) developed the median-effect principle based on the first models of Loewe (10) and Bliss (11). However, the median-effect principle is based on the mass-action law and not on statistics, and provides a diagnostic plot, including an isobologram. The median drug-effect equation is based on the four equations of Henderson-Hasselbach (19), Michaelis-Menten (20), Hill (21) and Scatchard (22), and therefore the dose-response curves of the single drug and combinations should be sigmoidal. Curves are plotted based on the doses of a drug (D1 and D2), and the effect of a drug, expressed as fraction affected $(\mathrm{Fa})$ and fraction unaffected $(\mathrm{Fu})$. An $\mathrm{Fa}$ of 0.5 means $50 \%$ growth inhibition, and $\mathrm{Fa}$ of 0.75 means $75 \%$ 
growth inhibition (or $25 \%$ growth); this means that $\mathrm{Fa}+\mathrm{Fu}=1$. The effect of a drug can be described as the ratio between $\mathrm{Fa} / \mathrm{Fu}=\left(\mathrm{D} / \mathrm{D}_{m}\right)^{m}$. Log transformation yields the median-effect equation: $\log (\mathrm{Fa} / \mathrm{Fu})=m \log (\mathrm{D})-m \log \left(\mathrm{D}_{m}\right)$, in which $\mathrm{D}$ is the dose, $m$ the sigmoidicity of the curve and $\mathrm{D}_{m}$ equals the dose that results in an $\mathrm{Fa}$ of 0.5 (i.e. $\mathrm{IC}_{50}$ ). By transforming $\mathrm{Fa}+\mathrm{Fu}=1$ to $\mathrm{Fu}=(1-\mathrm{Fa})$, the formula can be transformed into $\mathrm{Fa} /(1-\mathrm{Fa})=\left(\mathrm{D} / \mathrm{D}_{m}\right)^{m}$, and assuming $\mathrm{m}=1$ to $\mathrm{Fa}=\left[1+\left(\mathrm{D}_{m} / \mathrm{D}\right)\right]^{-1}$. When the effects for two drugs are combined and the formula for the CI is derived: $C I=[(D) 1 /(D 1-F a) 1]+[(D) 2 /(D 1-$ $\mathrm{Fa}) 2]+[\alpha(\mathrm{D}) 1(\mathrm{D}) 2 /(\mathrm{D} 1-\mathrm{Fa}) 1(\mathrm{D} 1-\mathrm{Fa}) 2]$, in which (D)1 and (D) 2 are doses used for combination, (D1-Fa)1 and (D1-Fa) 2 are the doses of the individual drugs resulting in $1-\mathrm{Fa}$; the slope is $m$, while $\alpha=1$ for mutually nonexclusive drugs. A CI above 1.2 is considered to be antagonistic, between 0.8 and 1.2 additive, and below 0.8 synergistic.

The usual presentation of the results requires a normal growth-inhibition curve and a plot of CI versus Fa (Figure 1D). The growth-inhibition curves are essential for proper evaluation, since it should be determined whether the curves are sigmoidal or hyperbolic, whether there are outliers, and whether sigmoidicity is similar for each drug. A simple quality check includes the comparison of the $\mathrm{D}_{m}$ value calculated by the program with the $\mathrm{IC}_{50}$ value from the curve, which should be similar. The $\mathrm{CI}-\mathrm{Fa}$ plot evaluates synergism over the whole Fa range from 0-1. However, CI values above an $\mathrm{Fa}$ of 0.95 are usually not reliable, while CI values obtained at $\mathrm{Fa}<0.5$ can be considered as not relevant, since $\mathrm{Fa}<0.5$ represent minor, clinically not relevant growth inhibition. In order to combine the data of more experiments, it is recommended to calculate the average of the CI values at $\mathrm{Fa}$ of $0.5,0.75$ and 0.90 for each separate experiment. Subsequently, the averages of each experiment can be used to calculate the means and SEM for the experiments. In this model, it should be specified whether the combined drugs act on a common target or have a similar mechanism of action, i.e. are mutually exclusive, where one agent may prevent the action of the other. The agents may have different mechanisms of action or targets and therefore act in a mutually nonexclusive manner.

In order to digitalize the median-effect method, the CalcuSyn software was designed based on the formulas given above by Chou and Talalay, allowing the user to plot the doseresponse curves of the single agents and the combination treatment to determine the CI. CalcuSyn can be downloaded from http://www.biosoft.com/w/calcusyn.htm, however a fee dependent on the intended number of users is required. In 2005, Chou published another software program based on the median-effect of the mass-action law, CompuSyn, which can be downloaded free of charge from www.combosyn.com. The program does not allow values above 1 or below 0 to be entered, which can be solved by plotting these values all at either 0.95 or 0.05 , respectively. Although this limitation does not allow evaluation of cell kill, this can be solved by using a modified calculation of $\mathrm{Fa}$ as described by Bijnsdorp et al. (8). Even though the program advises combining drugs at a fixed ratio based on the $\mathrm{IC}_{50}$, the current version also allows evaluation of non-fixed ratios. Loewe synergy using surfacearea models can be calculated using Combenefit, which can be downloaded for free from https://sourceforge.net/projects/ combenefit/.

Models evaluating the effect of two agents combined are valuable in the development of novel treatment strategies. Nevertheless, researchers should be aware of the limitations and pitfalls that these models carry. A concise summary of the advantages and disadvantages of the aforementioned methods are listed in Table I.

\section{How Should Novel Tyrosine Kinase-directed Drugs Be Combined?}

The models described above were developed primarily to evaluate combinations of two or more conventional anticancer chemotherapeutics. This raises the question of whether the model can also be used to evaluate combinations of conventional chemotherapy agents with novel chemotherapeutics drugs, such as tyrosine kinase inhibitors (TKI) or combinations of TKIs. There is sufficient evidence in literature that Calcusyn can be used to evaluate combinations of drugs such as pemetrexed or gemcitabine with a TKI, such as erlotinib or crizotinib, respectively (23, 24). However, for these studies, it is even more evident that genetic properties of the cells should be taken into account, while the concentration of a TKI should be chosen in a range high enough to modulate its target. For instance, in the case of erlotinib, it is not sensible to combine these drugs for tumours that have a $R A S$ mutation (23), while for crizotinib, either a MET proto-oncogene receptor tyrosine kinase ( $c$ $M E T$ ) amplification or mutation is required to achieve an effect (24). This also means that the drugs can better be combined at a non-fixed ratio, for which the current version of CalcuSyn has a separate option.

Similarly, combinations of one or more TKIs can be evaluated using the above-mentioned programs on the condition that genetic and biochemical properties are taken into account when combining these drugs. An example is the combination of erlotinib, an epidermal growth factor receptor (EGFR) inhibitor, with crizotinib, which inhibits c-MET. Resistance to erlotinib can be due to increased c-MET signalling, which can be inhibited by crizotinib. This combination appeared to be synergistic in cells with active EGFR and c-Met signalling, and additive when one of the pathways was active. Antagonism was observed in a cell line lacking these properties (25).

These considerations indicate that a lower concentration of one drug may be sufficient for modulating cellular signalling. 
Table I. Methods for determination of combination effects and their advantages and disadvantages.

\begin{tabular}{|c|c|c|}
\hline Method & Advantages & Disadvantages \\
\hline Fractional-effect analysis & $\begin{array}{l}\text { Straightforward method for mutually } \\
\text { non-exclusive drugs }\end{array}$ & $\begin{array}{l}\text { Model assumes linear concentration-effect } \\
\text { curve in contrast to reality (sigmoidal) } \\
\text { No confidence interval quantification }\end{array}$ \\
\hline Isobologram & Simple and accurate method & $\begin{array}{l}\text { Level of synergy or antagonism cannot be determined } \\
\text { The isobole based on the dose pair is often curvilinear } \\
\text { instead of linear due to variability in drug potency } \\
\text { No experimental variation quantification }\end{array}$ \\
\hline The response surface approach & $\begin{array}{l}\text { Model assumes sigmoidal } \\
\text { concentration-effect relationship }\end{array}$ & $\begin{array}{l}\text { Output interpretation requires expertise } \\
\text { since it is aggregated } \\
\text { Sufficient statistical knowledge essential }\end{array}$ \\
\hline Median drug-effect analysis & $\begin{array}{l}\text { A distinction between mutually exclusive } \\
\text { and non-exclusive acting agents can be made }\end{array}$ & $\begin{array}{l}\text { Synergy evaluation can be complicated } \\
\text { Fa values }<0 \text { or }>1 \text { cannot be included in calculations, } \\
\text { but by adapting the model this can still be achieved ( } 8 \text { ) }\end{array}$ \\
\hline
\end{tabular}

Fa: Fraction affected.

This has led some investigators to use ultra-low concentrations in the 'homeopathic' range, arguing that patient toxicity might also be reduced. However, when no clear effect on signalling is observed in mechanistic experiments, such combinations should be considered with care.

\section{Translation of Combinations to In Vivo Models}

When combining drugs in vivo, additional aspects should be taken care of. Ideally a synergistic effect on the tumour would be associated with an antagonistic effect on normal tissues. Unfortunately, this is not often observed and initial dosing in vivo (usually mice) should be done with care; moreover, the outcome often cannot be predicted with current in vitro alternative models, including organoids, since the whole in vivo mechanism of drug absorption, distribution, metabolism and excretion is not present therein. Usually one of these aspects can be investigated in a proper model (e.g. cytochrome p450 (CYP)-mediated metabolism or glucuronidation and other phase I and II metabolic pathways or ATP-binding cassette transporter-mediated efflux) and can be used to adapt the in vivo scheduling. Therefore, it is advised to start with a lower dose of at least one of the drugs. Usually it is advised to give one drug at the maximal tolerated dose (MTD) of the single agent and the other at its $0.66 \times$ MTD, although most investigators start with each drug at its $0.66 \times$ MTD. Serial two-fold dilutions can be tested before an efficacy experiment in case severe toxicity is expected. In vitro experiments can provide guidelines on the scheduling for animal experiments, but toxicity might also be synergistic, necessitating alternative scheduling. Mechanistic pharmacological studies in animals will provide essential information on proper dosing and scheduling in vivo. The above-mentioned example of gemcitabine and cisplatin showed pre-treatment of cell lines with gemcitabine would increase the formation of DNA- platinum adducts and prevent repair of these adducts. However, dosing for both drugs had to be reduced, but the combined effect was superior to that of each drug alone at its MTD (26). The same principle was applied to the combination of gemcitabine and crizotinib in mice, in which the combination was based on in vitro synergism in a pancreatic cancer cell line with a $c-M E T$ amplification. It was decided to give gemcitabine at its $0.83 \times$ MTD. The combination of gemcitabine and crizotinib was superior to that of each drug separately, while crizotinib increased the accumulation of the active metabolite of gemcitabine by inhibition of gemcitabine degradation (24). In short, it can be concluded that for in vivo combinations, the schedule can often be deduced from in vitro data, while dosing should be reduced slightly.

\section{New Computational-based Approaches for Capturing Cellular and Signalling Complexity}

The rapid development and application of machine-learning methods is now also being applied in biomedical sciences. The utilisation of several machine-learning methods for assessment of combination drug therapy for HIV, hypertension, infectious diseases and cancer is described elaborately by Tsigelny (27). For example, machine-learning networks can implement various parameters, such as i) compound-specific physical and chemical properties; ii) biochemical response of target molecule(s); iii) cellular response; and iv) patient characteristics, including genomic, proteomic and metabolomic profiles, which sounds promising.

Current predictive models for drug-combination effects are commonly based on high-throughput testing of drug combinations for each cell line. These data are then used to identify the molecular features that predict therapy response (28). A pan-cancer DREAM community challenge, the 
AstraZeneca Drug Combination Prediction Challenge, showed such findings on a pan-cancer scale (29). Synergy prediction based on models of drug interactions are appearing.

Given that most therapy combination approaches are based on an aggregated index of synergy, the analytical methods that exist today might need additional refinements to capture intercellular (cell identity) as well as intracellular heterogeneity (signalling activity) and their combined effects on efficacy of drug combinations. Single-cell tracing methods, such as single-cell genetic/mRNA profiling, fluorescent reporter systems and cell-tracing barcode technologies can provide relevant insight into population changes as well as fluctuations in the mechanism of action that result in therapy efficacy. These computational models should provide sufficient complexity to predict the effect of combination therapies on a cellular/signalling level accurately. Given this high complexity, more advanced machine-learning methods, such as deep learning, might be needed to enable adequate modelling.

\section{Conclusion}

Drug combinations have been used for decades in the clinic to enhance the treatment of patients, and are therefore not a novelty in the field of pharmacology. Various in vitro models together with mathematical equations based on the Bliss independence model, Loewe additivity model or a combination of both, i.e. the median drug effect, enable the prediction of the effect of two agents combined. Nonetheless, application of these models should be tailored to the context of each study and with awareness of the limitations and advantages of each method. Considering the increment in the development of novel therapeutics, the number of combinations that can be made is substantial. Aside from more complex single-cell assay read-outs that are able to capture cellular and signalling heterogeneity, more advanced computational models might be needed, including deeplearning methodologies. Therefore, we anticipate that refinements of the classical synergy models with emerging artificial intelligence-based models will benefit the investigation of new combination treatments in the near future.

\section{Conflicts of Interest}

The Authors have no conflicts of interest to declare in regard to this study.

\section{Authors' Contributions}

$\mathrm{BEH}, \mathrm{GJ}, \mathrm{BW}$ and EG wrote the article. GM provided the figures and GLP the table. MJ, LB, HNW, AVM and MCMK revised the article.

\section{Acknowledgements}

This work was partially supported by the following grants: CCA Foundation 2012 and 2015 grants (GJP, EG), Fondazione Pisana per la Scienza (EG), KWF Dutch Cancer Society grants (KWF project\#10401 and \#11957, EG) and AIRC/Start-Up grant (EG).

\section{References}

1 DeVita VT and Chu E: A history of cancer chemotherapy. Cancer Res 68(21): 8643-8653, 2008. PMID: 18974103. DOI: 10.1158/0008-5472.CAN-07-6611

2 Bertino JR and Chou T-C: Chemotherapy: synergism and antagonism. In: Encyclopedia of Cancer. Vol 1. Academic Press, Inc., pp. 368-379, 1997.

3 Peters GJ, Van Der Wilt CL, Van Moorsel CJA, Kroep JR, Bergman AM and Ackland SP: Basis for effective combination cancer chemotherapy with antimetabolites. Pharmacol Ther 87(2-3): 227-253, 2000. PMID: 11008002. DOI: 10.1016/S01637258(00)00086-3.

4 Bergman AM, Ruiz Van Haperen VWT, Veerman G, Kuiper CM and Peters GJ: Synergistic interaction between cisplatin and gemcitabine in vitro. Clin Cancer Res 2(3): 521-530, 1996. PMID: 9816199. DOI: 10.1016/j.canlet.2011.12.016

5 Peters GJ, Backus HH, Freemantle S, Van Triest B, CodacciPisanelli G, Van der Wilt CL, Smid K, Lunec J, Calvert AH, Marsh S, McLeod HL, Bloemena E, Meijer S, Jansen G, Van Groeningen CJ and Pinedo HM: Induction of thymidylate synthase as a 5-fluorouracil resistance mechanism. Biochim Biophys Acta Mol Basis Dis 1587(2-3): 194-2015, 2002. PMID: 12084461. DOI: 10.1016/S0925-4439(02)00082-0

6 Bergman AM, Pinedo HM and Peters GJ: Steroids affect collateral sensitivity to gemcitabine of multidrug-resistant human lung cancer cells. Eur J Pharmacol 416(1-2): 19-24, 2001. PMID: 11282108. DOI: 10.1016/S0014-2999(01)00858-5

7 Perego P, Hempel G, Linder S, Bradshaw TD, Larsen AK, Peters GJ and Phillips RM: Cellular pharmacology studies of anticancer agents: recommendations from the EORTC-PAMM group. Cancer Chemother Pharmacol 81(3): 427-441, 2018. PMID: 29285635. DOI: $10.1007 / \mathrm{s} 00280-017-3502-7$.

8 Bijnsdorp IV, Giovannetti E and Peters GJ: Analysis of drug interactions. Methods Mol Biol 731: 421-34, 2011. PMID: 21516426. DOI: 10.1007/978-1-61779-080-5_34

9 Peters GJ, Chatelut E, Larsen AK and Zaffaroni N: EORTCrelated new drug discovery and development activities: Role of the Pharmacology and Molecular Mechanisms Group. Eur J Cancer Suppl 10(1): 128-140, 2012. DOI: 10.1016/S1359-6349 (12)70022-8

10 Loewe S and Muischnek H: Effect of combinations: mathematical basis of problem. Arch Exp Pathol Pharmacol 114: 313-326, 1926.

11 Bliss CI: The toxicity of poisons applied jointly. Ann Appl Biol 26: 585-615, 1939. DOI: 10.1111/j.1744-7348.1939.tb06990.x

12 Webb J: Effect of more than one inhibitor. Enzymes Metabol Inh 1: 66-79, 1963.

13 Elion GB, Singer S and Hitchings GH: Antagonists of nucleic acid derivatives. VIII. Synergism in combinations of biochemically related antimetabolites. J Biol Chem 208(2): 477488, 1954. PMID: 13174557.

14 Steel GG and Peckham MJ: Exploitable mechanisms in combined radiotherapy-chemotherapy: The concept of additivity. 
Int J Radiat Oncol Biol Phys 5(1): 85-91, 1979. PMID: 422420. DOI: 10.1016/0360-3016(79)90044-0

15 Chou T and Talalay P: Quantitative dose-effect relationships: The combined effects of multiple. Adv Enzyme Regul 22: 27-55, 1984. PMID: 6382953. DOI: 10.1016/0065-2571(84)90007-4

16 Berenbaum MC: What is synergy? Pharmacol Rev 41(2): 93141, 1989. PMID: 2692037.

17 Di Veroli GY, Fornari C, Wang D, Mollard S, Bramhall JL, Richards FM and Jodrell DI: Combenefit: An interactive platform for the analysis and visualization of drug combinations. Bioinformatics 32(18): 2866-2868, 2016. PMID: 27153664. DOI: $10.1093 /$ bioinformatics/btw230

18 Greco WR, Faessel H and Levasseur L: The search for cytotoxic synergy between anticancer agents: A case of Dorothy and the ruby slippers? J Natl Cancer Inst 88(11): 699-700, 1996. PMID: 8637018. DOI: $10.1093 / \mathrm{jnci} / 88.11 .699$

19 Clark WM: The Determination of Hydrogen Ions. Third edition. Baltimore, Williams and Wilkins, 1928.

20 Michaelis L and Menten ML: Die Kinetik der Invertinwirkung. Biochem Z 49: 333-369, 1913.

21 Hill AV: The Combinations of haemoglobin with oxygen and with carbon monoxide. I Biochem J 7: 471-480, 1913.

22 Scatchard G and George: The attractions of proteins for small molecules and ions. Ann N Y Acad Sci 51: 660-672, 1949.

23 Giovannetti E, Lemos C, Tekle C, Smid K, Nannizzi S, Rodriguez JA, Ricciardi S, Danesi R, Giaccone G and Peters GJ: Molecular mechanisms underlying the synergistic interaction of erlotinib, an epidermal growth factor receptor tyrosine kinase inhibitor, with the multitargeted antifolate pemetrexed in nonsmall-cell lung cancer cells. Mol Pharmacol 73(4): 1290-1300, 2008. PMID: 18187583. DOI: 10.1124/mol.107.042382

24 Avan A, Caretti V, Funel N, Galvani E, Maftouh M, Honeywell RJ, Lagerweij T, Van Tellingen O, Campani D, Fuchs D, Verheul HM, Schuurhuis GJ, Boggi U, Peters GJ, Würdinger T and Giovannetti E: Crizotinib inhibits metabolic inactivation of gemcitabine in c-Met-driven pancreatic carcinoma. Cancer Res 73(22): 6745-6756, 2013. PMID: 24085787. DOI: 10.1158/000 8-5472.CAN-13-0837

25 Van Der Steen N, Leonetti A, Keller K, Dekker H, Funel N, Lardon F, Ruijtenbeek R, Tiseo M, Rolfo C, Pauwels P, Peters GJ and Giovannetti E: Decrease in phospho-PRAS40 plays a role in the synergy between erlotinib and crizotinib in an EGFR and cMET wild-type squamous non-small cell lung cancer cell line. Biochem Pharmacol 166: 128-138, 2019. PMID: 31078602. DOI: $10.1016 /$ j.bcp.2019.05.014
26 Van Moorsel CJA, Pinedo HM, Smid K, Comijn EM, Voorn DA, Veerman G, Lakerveld B, Van der Vijgh WJF, Giaccone G, Postmus PE and Peters GJ: Schedule-dependent pharmacodynamic effects of gemcitabine and cisplatin in mice bearing Lewis lung murine non-small cell lung tumours. Eur J Cancer 36(18): 2420-2429, 2000. PMID: 11094319. DOI: 10.1016/ S0959-8049(00)00345-2

27 Tsigelny IF: Artificial intelligence in drug combination therapy. Brief Bioinform, 2018. PMID: 29438494. DOI: 10.1093/ bib/bby004

28 Bansal M, Yang J, Karan C, Menden MP, Costello JC, Tang H, Xiao G, Li Y, Allen J, Zhong R, Chen B, Kim M, Wang T, Heiser LM, Realubit R, Mattioli M, Alvarez MJ, Shen Y, Gallahan D, Singer D, Saez-Rodriguez J, Xie Y, Stolovitzky G, Califano A, Abbuehl JP, Altman RB, Balcome S, Bell A, Bender A, Berger B, Bernard J, Bieberich AA, Borboudakis G, Chan C, Chen TH, Choi J, Coelho LP, Creighton CJ, Dampier W, Davisson VJ, Deshpande R, Diao L, Di Camillo B, Dundar M, Ertel A, Goswami CP, Gottlieb A, Gould MN, Goya J, Grau M, Gray JW, Hejase HA, Hoffmann MF, Homicsko K, Homilius M, Hwang W, Ijzerman AP, Kallioniemi O, Karacali B, Kaski S, Kim J, Krishnan A, Lee J, Lee YS, Lenselink EB, Lenz P, Li L, Li J, Liang H, Mpindi JP, Myers CL, Newton MA, Overington JP, Parkkinen J, Prill RJ, Peng J, Pestell R, Qiu P, Rajwa B, Sadanandam A, Sambo F, Sridhar A, Sun W, Toffolo GM, Tozeren A, Troyanskaya OG, Tsamardinos I, Van Vlijmen HWT, Wang W, Wegner JK, Wennerberg K, Van Westen GJP, Xia T, Yang Y, Yao V, Yuan Y, Zeng H, Zhang S, Zhao J and Zhou J: A community computational challenge to predict the activity of pairs of compounds. Nat Biotechnol 32(12): 1213-1222, 2014. PMID: 25419740. DOI: 10.1038/nbt.3052

29 Menden MP, Casale FP, Stephan J, Bignell GR, Iorio F, McDermott U, Garnett MJ, Saez-Rodriguez J and Stegle O: The germline genetic component of drug sensitivity in cancer cell lines. Nat Commun 9(1): 3385, 2018. PMID: 30139972. DOI: $10.1038 / \mathrm{s} 41467-018-05811-3$
Received May 14, 2019

Revised June 7, 2019

Accepted June 12, 2019 